\title{
Electromagnetic configurable architectures for assessment of Carbon Fiber Reinforced Plastics
}

\author{
Rozina Steigmann ${ }^{1,2}$, Adriana Savin ${ }^{1, *}$, Nicoleta Iftimie $^{1}$, Gabriel Silviu Dobrescu ${ }^{1}$, Zoran \\ Bergant $^{3}$, and Janez Grum ${ }^{3}$ \\ ${ }^{1}$ National Institute of R\&D for Technical Physics, NDT Department, Iasi, Romania \\ ${ }^{2}$ Alexandru Ioan Cuza University, Faculty of Physics, Iasi, Romania \\ ${ }^{3}$ University of Ljubljana, Faculty of Mechanical Engineering, Ljubljana, Slovenia
}

\begin{abstract}
Carbon Fiber Reinforced Plastics are used in most wide domains due their low density, lack of mechanical fatigue phenomena and high strength -to weight ratio. From electromagnetic point of view, Carbon Fiber Reinforced Plastics structure represents an inhomogeneous structure of electric conductive fibers embedded into a dielectric material, thus an electromagnetic configurable architecture can be used to evaluate above mentioned defects. The paper proposes a special sensor, send receiver type and the obtaining of electromagnetic image by post-processing each coil signals in each point of scanning, using a sub-encoding image reconstruction algorithm and super-resolution procedures. The layout of fibers can be detected interrogating only diagonal reception coils.
\end{abstract}

\section{Introduction}

Carbon Fiber Reinforced Plastics (CFRP) started to be used as usually materials in aerospace industry since the middle of the last century [1]. Nowadays, AIRBUS A350 is 52\% CFRP, including wing spars and fuselage components [2]. The high costs of CFRP have been surpassed by their strength-to-weight ratio and feasibility. The CFRP structures in aviation/aerospace industry make compromise between advantages - weight reduction, the mechanical properties can be tailored by layout design, high resistance to corrosion at interfaces with metallic joints and disadvantages as high recurring costs, barely visible defects appearance and their propagation, difficulties in repairing, raising the requirement of testing during the fabrication of structures as well as in service monitoring. CFRP structures can be evaluated by many non-destructive techniques (NDT) using conventional strain gages [3], ultrasound using different types of sensors and methods [4,5], Bragg fibers [6], PZT sensors [7], thermography and acoustic emission [8,9] and also sensors for electrical properties, wireless sensing with low sampling rate, wireless strain and temperature sensors, especially knowing that carbon fibers have electrical properties $[10,11]$ and these properties are modified by the effect of impact with low energies [12].

Due to the numerous types of layups and layouts in configuring the composites in function of layers number, orientation of fibers and woven, type of matrix, etc., the full effect of design

\footnotetext{
* Corresponding author: asavin@phys-iasi.ro
} 
over the behavior of the composites at impact isn't yet elucidated, different designs leading to the development of different control methods [13, 14]. Taking into studies samples of CFRP, usually used in aerospace engineering, carbon fiber embedded in Polyphenylene sulphide (PPS) matrix, this paper present a novel type of electromagnetic sensors defined as CA due to the possibility to interrogate only a part of the sensors from the architecture or the entire assembly, to detect damages induced by low energy impact, increasing the spatial resolution compared to the classical sensors. The electromagnetic image is obtained by postprocessing each coil signals in each point of scanning, using a sub-encoding image reconstruction algorithm and super-resolution procedures. The novelty consist in the possibility to interrogate diagonal reception coils in order to detect the fiber breaks and delamination that are tilted from the direction of scanning. The interrogation is sequentially made by programming the acquisition data board. The method will be further developed for semi-automatic control of complex structures made from composites.

\section{Materials and methods}

\subsection{Studied samples}

The strength in using CFRP is given by the volume ratio of fibers and matrix. A good ratio means a qualitative composite. Plates of CFRP made by Tencate, the Netherlands having the dimensions $150 \mathrm{mmx} 100 \mathrm{mmx} 4.2 \mathrm{~mm}$ [15] have been taken into study. The reinforcement consists in 12 biaxial woven $\left[0^{\circ} / 90^{\circ}\right.$ ] harness satin 5H-T300JB type. The successive laminas are disposed by rotation at $45^{\circ}$, obtaining a quasi-isotropic material, the matrix is PPS. This offers exceptional chemical resistance at high temperatures, comparable to PEEK, along with excellent thermal properties for long-term (over $200^{\circ} \mathrm{C}$ ) and short-term use (up to $260^{\circ} \mathrm{C}$ ) [16]. The fiber volume ratio is $50 \pm 3 \%$ and the composite density is $\rho=1520 \mathrm{~kg} / \mathrm{m}^{3}$. This composite has been used in the construction of Airbus and Boeing aircrafts [17]. The properties of weave weft for input in simulation are yarn width $1.31 \mathrm{~mm}$, yarn thickness 0.162 $\mathrm{mm}$, yarn spacing $1.50 \mathrm{~mm}$, yarn filament count 3000, filament diameter $0.007 \mathrm{~mm}$, carbon fiber density $1.75 \mathrm{~g} / \mathrm{cm}^{3}[18]$. The microstructure of the cross-section was determined using microscope Olympus BX51 coupled with digital camera using QuickPhoto Micro3.1 in order to see that the material is very compact, having no voids or mislaying of tows. These kind of flaws can influence the mechanical behavior of the composite (Figure 1).
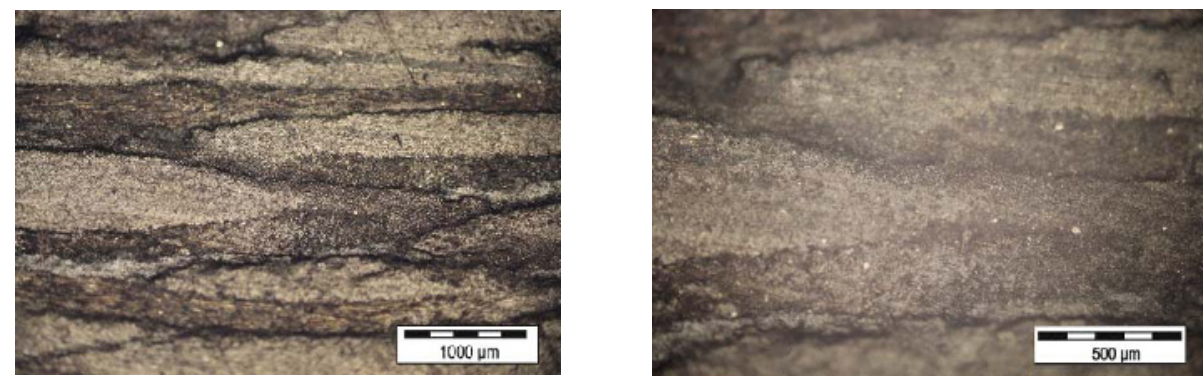

Fig. 1. Microstructure of the cross-section of the composite.

Using equipment FRACTOVIS PLUS 9350-CEAST-Instron USA [12], [15], the samples were impacted with different energies to obtain delamination. Figure 2a present the 5 Harness Satin woven layout and Figure $2 \mathrm{~b}$ shows the front side of impacted sample, at $10 \mathrm{~J}$ energy, which creates a concavity at surface and delamination inside. 

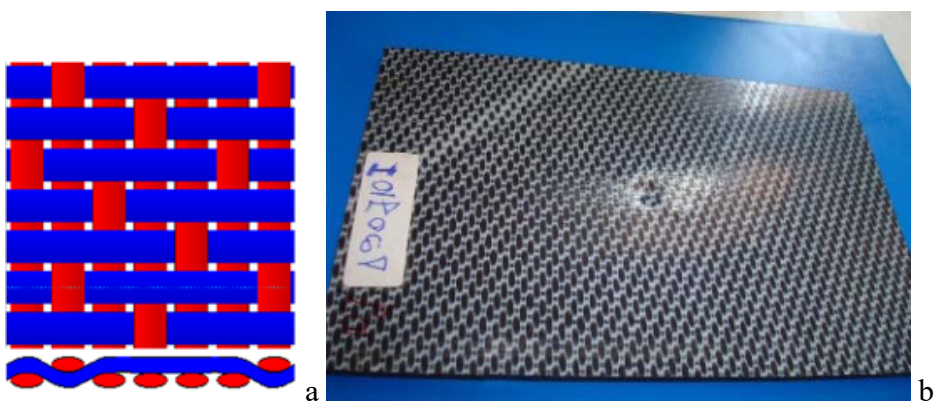

Fig. 2. Studied samples: a) $5 \mathrm{H}$ satin layout; b) front side of impacted sample.

\subsection{Sensor concept}

The sensor consists in a 2D architecture of 9 identical reception coils having inner radii $1 \mathrm{~mm}$ and outer radii $1.2 \mathrm{~mm}$, having 18 turns with $0.03 \mathrm{~mm}$ from CuEm placed into a polyimide support with $7 \times 7 \times 2 \mathrm{~mm}$ dimensions. The emission coil has 1 turn from $\mathrm{CuEm}$ wire with $1 \mathrm{~mm}$ diameter, has average side of $7.1 \mathrm{~mm}$ and is placed around the half height of the support. For CFRP plate with above characteristics; the moduli of the magnetic field components generated by a current through the emission coil at frequency of $300 \mathrm{kHz}$ and amplitude of $0.1 \mathrm{~A}$ is presented in Figure 3, in the middle, the field assures a uniform value. The design of the CA with 2D sensor array of receiver coils is presented in Figure 4.

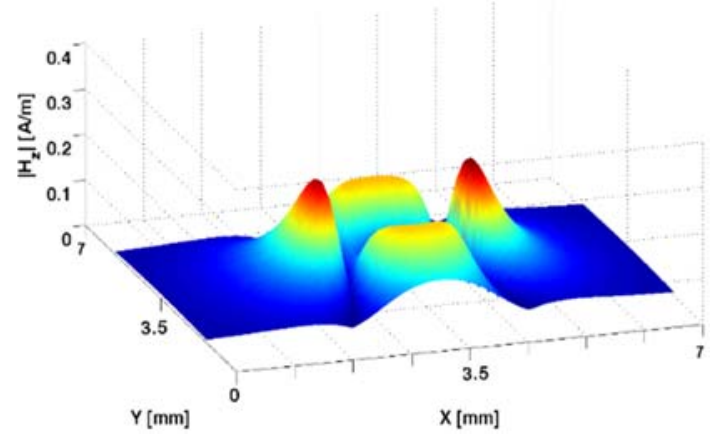

Fig. 3. Magnetic field provided by the emission coil.

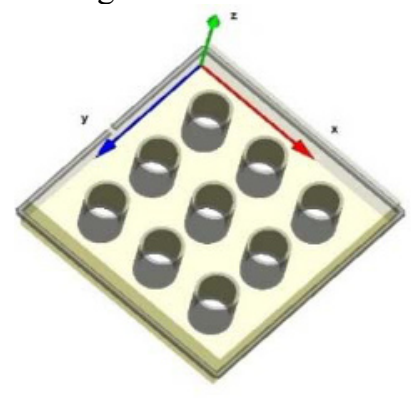

Fig. 4. Configurable architecture.

The geometry was tested based on simulation using Dyadic Green function and volume integral method described in $[19,20]$. The functioning of 2D sensor array [21] assures good lateral resolution and emphasizes hidden defects by simultaneous interrogation of the reception coils by means a multiplexor, improving the scan time and the response resolution. An internal multiplexing system reduces the mutual inductance and programs the actual time when each coil is interrogated. The architecture is configurable, being able to interrogate only few of reception coils by programming the data acquisition board.

The reception coils detect the reflections of electromagnetic field (EM) created by the emission part in composite, the system measuring the received signal both in amplitude and phase. The behavior of the CA has been simulated using XFDTD software, Remcom, placing a simulated delamination under the reception coils denoted $(3.2,3.3)$, Figure 5. Finite Difference Time Domain (FDTD) finds approximate solutions to Maxwell's equation in differential form, discretizing using central-difference by 30 approximations to the space and time partial derivatives.

The equations are implemented in the software and solved in a cyclic manner: the electric field vector components in a volume of space are solved in time, the magnetic field vector 
components in the same spatial volume are solved at the next instant in time. The process is repeated until the desired transient or steady-state EM field behavior is fully achieved. The FDTD method is able to cover a range of frequency in a single simulation using Fourier transform, treating the simulated structures with a wide variety of EM material properties. Both time domain and frequency domain information are offered for complete characterization of a lot of problems and applications in electromagnetics.

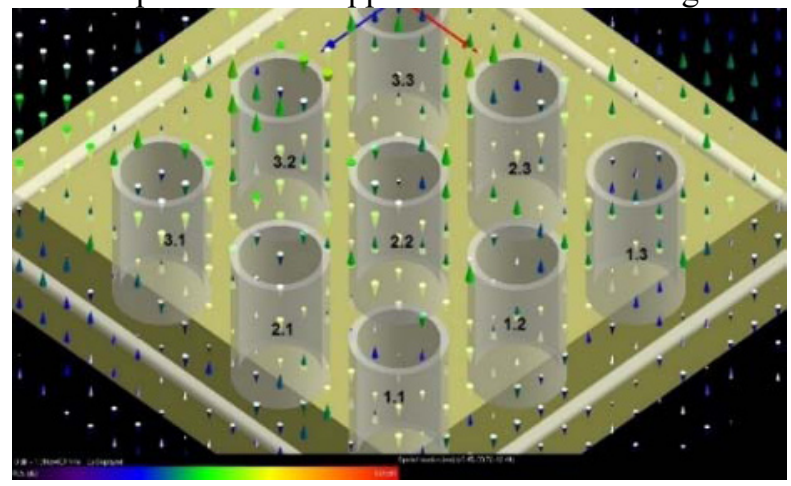

Fig. 5. FDTD simulation.

The 2D array allows parallel acquisition of the signals, reducing the data storage and processing requirements. The signals are reconstructed into separate electromagnetic images and can be improved using super-resolution (SR) procedures [21, 22]. SR is a technique in terms of its ability to resolve closely spaced scatters in a solid, measuring the near field of scattered waves. Using notations from [21] SR uses the maximum likelihood estimation procedure [23] for coherent sources. The source locations are located where the function $\left(\bar{S}^{*} \bar{R}_{x x}\right)$ is maximal, where $\bar{R}_{x x}$ is the autocorrelation matrix of sensor signal response. The SR method can lead to improvement of the signal to noise region (SNR). The SNR in dB was measured in the time domain $S N R=20 \log _{10} A_{\text {signalpeak }} / A_{\text {noiseRMS }}$ where $A_{\text {signalpeak }}$ represent the peak amplitude of signal reflected of the discontinuities and $A_{\text {noise }}$ MS represent the RMS of noise measured away of the signal. The array gain $\mathrm{G}$ is defined as $G=S N R_{\text {array }} / S N R_{\text {sensor }}$ and here $\mathrm{G}=0.7 * \mathrm{~N} * \mathrm{M}$, where $M$ and $N$ represent the number of rows and respectively columns of reception coils from the array. Applying SR method on the results obtained by numerical simulation, Figure 6, the sensor array response is obtained (Figure 7).

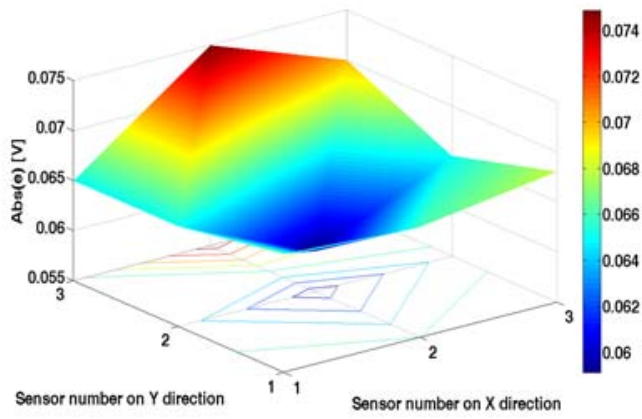

Fig. 6. Amplitude of the signal on each of the 9 receiver coils placed on a simulated delamination.

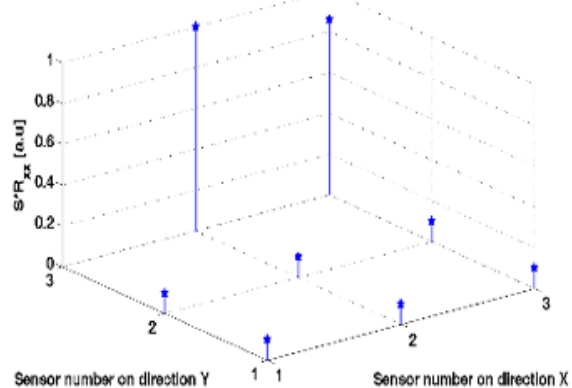

Fig.7. Simulation of signals delivered by the sensors from array, using the SR procedure.

The maximum value corresponds to position where the delamination is placed under the coils indexed $(3.2,3.3)$. 


\subsection{Experimental set-up}

The experimental set-up is presented in Figure 8. The emission coil is fed through a function generator WW1074 Tabor Ltd, the signal is amplified by a power amplifier RF A1012 AGT\&C Power Conversion Inc. The multiplexer send the received signal to the data acquisition towards PC.

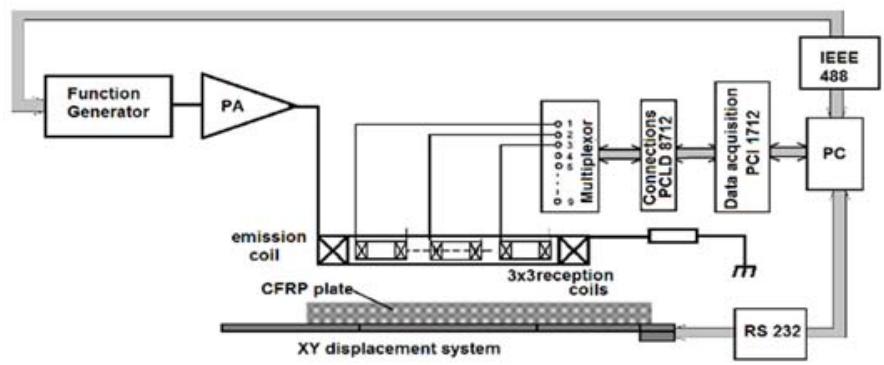

Fig. 8. Experimental set-up.

The samples to be examined are displaced under the sensor, being raster scanned with a $\mathrm{XY}$ automated displacement system, Newmark USA, the acquisition of signal being synchronized with the buffering of it. The experimental devices are controlled by PC though algorithms developed in Matlab 2014a.

\section{Results and discussions}

The answer of sensor array for experimental measurements of CFRP of the plate with impacted zone is presented in Figure 9a after the signal processing. The sensor array is placed in the same region for which the simulation has been carried on.
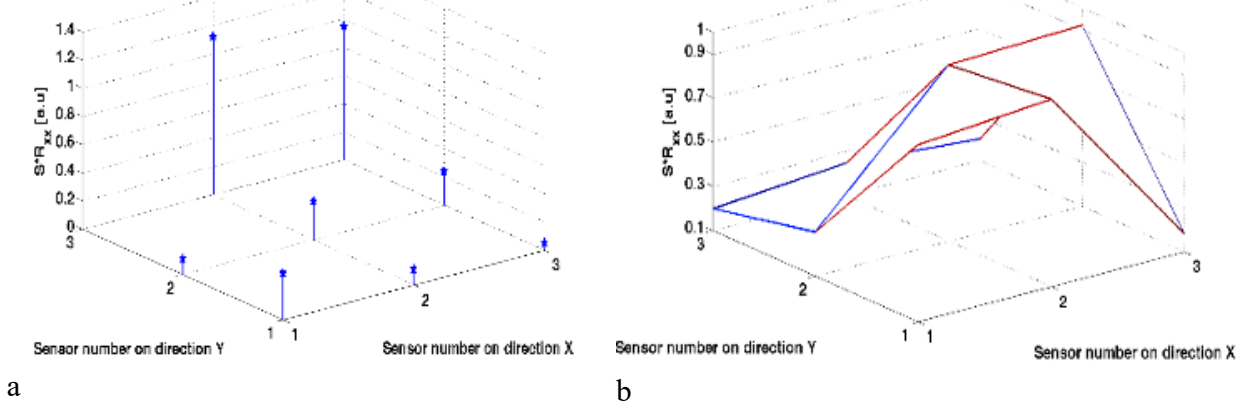

Fig. 9. a) Signals delivered by the sensors from array, using the super-resolution procedure; b) signals received when the $45^{\circ}$ fibers are displaced by delamination.

Comparing the results from Figure 7 with Figure 9a, it can be observed that the position of maxima are clearly improved for experimental data. Figure $9 \mathrm{~b}$ presents the response of the sensor array using the SR method for signal processing, in the region where the position of carbon fibers at $45^{\circ}$ have been disturbed from the layout. A surface of $60 \times 60 \mathrm{~mm}$ of the composite is scanned using above mentioned system. The EM image [24] of the surface is obtained by post-processing the acquired signals in each coil in each point of scan using a sub encoding image reconstruction algorithm. A sub encoding image reconstruction starts at the beginning scanning point with the set of component signals received using a reduced number of phase-encoding gradient steps. Fourier transformation of these signal sets results 
in aliased component images. Further, the sub encoding reconstruction operates entirely in the image domain based on the fact that each pixel in an aliased image is a superposition of multiple pixels [25]. The results obtained at the reading of the middle columns sensors, as numbered in Figure 5, are presented in Figure 10. The method allows the visualization of delamination induced in the composite plate due to impact with low energy.

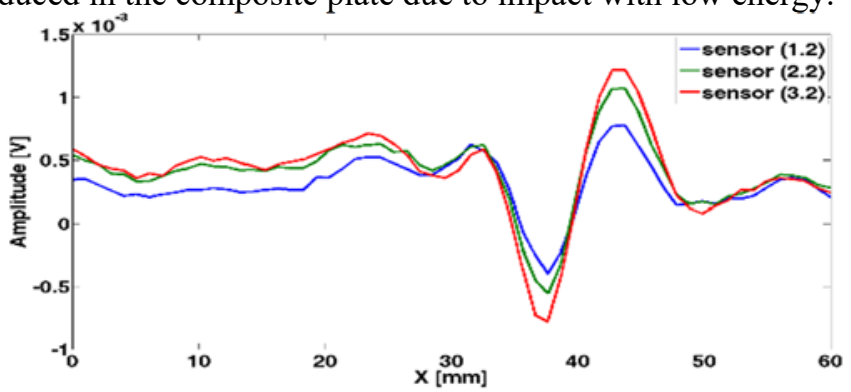

Fig. 10. Signals recorded by the middle column sensors.

In Figure 11a is presented the electromagnetic image of the plate impacted with $10 \mathrm{~J}$. It can be observed the area of delamination and the breaks of few fibers. Interrogating only the diagonal reception coils, the layout of $45^{0}$ fiber orientation can be also emphasized. The results are presented in Figure 11b.
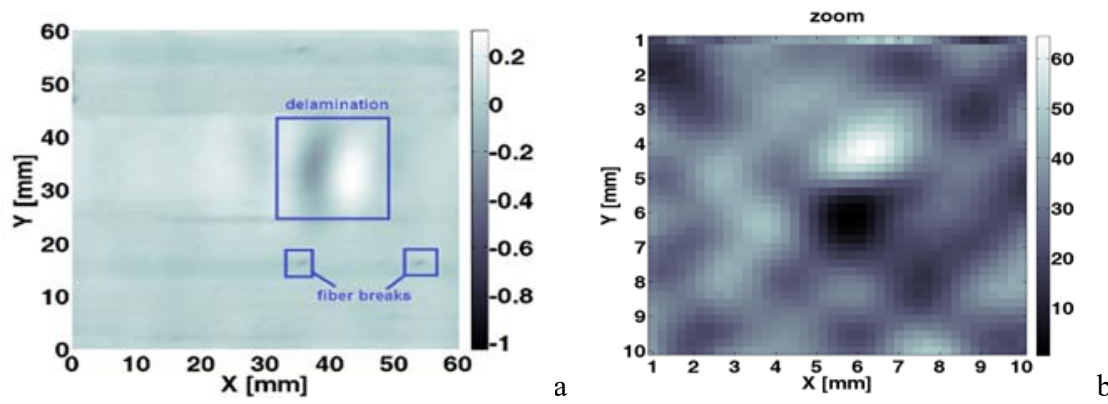

Fig. 11. Electromagnetic image: a) entire scanned region; b) zoom on 10x10mm around the central region of the delamination.

\section{Conclusions}

Using a suitable CA of sensors and the SR procedures as the signal post processing method, will lead to improvement of the SNR reported to the situation in which only one sensor is used. The simulations and their validations using real measurements prove the ability of the $\mathrm{CA}$ of sensors to improve the resolution of control. Also, the evaluation become more efficient, the scanning time being reduced and parallel acquisition of the signals digitized and recorded, reduces the data storage and processing requirements. Delamination's due to impact with low energies can be emphasized and localized, as well as displacement of the fibers from the layout are determined. The electromagnetic image is obtained by postprocessing each coil signals in each point of scanning, using a sub-encoding image reconstruction algorithm and super-resolution procedures. The layout of fibers can be detected interrogating only diagonal reception coils. The method can be applied for rapid detection of flaws or misalignment of fibers/layers after a certain functioning time in any domain where CFRP composites are employed, especially for semi-automatic control of complex components made from CFRP. 
Further tests for curve surfaces can be made embedding the sensors on a flexible support, allowing the enveloping of a surface as turbine blades or corners and joints of composite products.

This paper is partially supported by Romanian Ministry of Research and Innovation under project PNII-PCCA-2013-4-0656 and Nucleus Program PN 16-37 0101.

\section{References}

1. K. Potter, An Introduction to Composite Products: Design, Development and Manufacture (Springer, 5th Ed. Chapman \& Hall, London 1996)

2. G. Marsh, Reinforced plastics 54, 6, 20-24 (2010)

3. R. Steigmann, A. Savin, V. Goanta, P. D. Barsanescu, B. Leitoiu, N. Iftimie, M D. Stanciu, I. Curtu, IOP Conf. Ser. Mat. Sci. 147, 012140 (2016)

4. R.Grimberg, A. Savin, R. Steigmann, A. Bruma, P.D. Barsanescu, P. D. Salavastru, Proceedings of NDT in Progress 2009, 65-74 (2009)

5. A.A. Karabutov, N.B. Podymova, I.O. Belyaev, Acoustical Physics 59, 6, 667-673 (2013)

6. G. Luyckx, E. Voet, W. De Waele, J. Degrieck, Smar. Mat. St. 19, 10, 105017 (2010)

7. G. Dib, E. Koricho, O. Karpenko, M. Haq, L. Udpa, AIP Conference 1650, 1, 1072-1080 (2015)

8. J. Kober, Z.Prevorovsky, M. Chlada, Sensors and Actuators A: Physical, 240, 50-56 (2016)

9. E.Z. Kordatos, D.G. Aggelis, T.E Matikas, Compos. Part B: Eng. 43, 6, 2676-2686 (2012)

10. R. Grimberg, D. Premel, A. Savin, Y. Le Bihan, D. Placko, Insight 43, 4, 260-264 (2001)

11. Y. He, G. Tian, M. Pan, D. Chen, Compos. Struct.109, 1-7 (2014)

12. R. Steigmann, A. Savin, Advanced Sensor for Enhancement of Electromagnetic Imaging of Impacted, Carbon Fibers-Pps Composites, Chapt. 52 in DAAAM International Scientific Book, 633-644 (Katalinic, B., Ed.; DAAAM International: Vienna, Austria, 2014)

13. W. Yin, P.J. Withers, U. Sharma, A.J .Peyton, IEEE T.Instrum. Meas. 58, 3, 738-743 (2009)

14. G. Mook, J. Pohl, F. Michel, Smart Mat. Struct, 12, 6, 997-1004 (2003)

15. A. S. T. M. D7136, Standard test method for measuring the damage resistance of a fiberreinforced polymer matrix composite to a drop-weight impact event (2005)

16. S.Zhou, Q. Zhang, C. Wu, J. Huang, Mater. Design, 44, 493-499 (2013)

17. J. Chacksfield, Aircraft Design, Systems and Operations Meeting (1987)

18. S. Daggumati, E. Voet, W. Van Paepegem, J. Degrieck, J. Xub, S.V. Lomov, I. Verpoest, Compos. Sci. Technol. 71, 1171-1179 (2011)

19. O. Mihalache, R. Grimberg, E. Radu, A. Savin, Sensor.Actuat. A: Phys., 59, 1-3 (1997).

20. E. Radu, R. Grimberg, A. Savin, O. Mihalache, Sensor.Actuat. A: Phys. 59, 1, 213-218 (1997)

21. R. Grimberg, L. Udpa, A. Savin, R. Steigmann, NDT \& E Int, 39 (4), 264-271 (2006)

22. S.V. Shell, W.A. Gardner, High resolution direction finding (Bose NK, Rao CR, editors. Handbook of statistics, vol.10. Amsterdam: Elsevier; 1993)

23. L.Zhao, P. Krishnaiah, Z. Boi, J. Mutivariate Anal, 20, 1-25 (1986)

24. http://www.solvay.com/en/binaries/Composites-Specialty-Polymers_EN-220508.pdf

25. D. K.Sodickson, Spatial encoding using multiple RF coils: SMASH imaging and parallel MRI, eMagRes (2000). 